\title{
Stochastic Resonance in Neuron Models: Endogenous Stimulation Revisited
}

\author{
Hans E. Plesser ${ }^{1,2, *}$ and Theo Geisel ${ }^{1}$ \\ ${ }^{1}$ Max-Planck-Institut für Strömungsforschung and Fakultät für Physik, Universität Göttingen \\ Bunsenstraße 10, 37073 Göttingen, Germany \\ ${ }^{2}$ Institutt for tekniske fag, Norges landbrukshøgskole, P.O.Box 5065, Ås, Norway
}

(To appear in Phys Rev E)

The paradigm of stochastic resonance (SR) - the idea that signal detection and transmission may benefit from noisehas met with great interest in both physics and the neurosciences. We investigate here the consequences of reducing the dynamics of a periodically driven neuron to a renewal process (stimulation with reset or endogenous stimulation). This greatly simplifies the mathematical analysis, but we show that stochastic resonance as reported earlier occurs in this model only as a consequence of the reduced dynamics.

87.19.La, 05.40.-a

\section{INTRODUCTION}

The improvement of signal transmission and detection through noise has been studied keenly over the past two decades under the paradigm of stochastic resonance; for recent reviews see Refs. [1,2]. This holds true for the neurosciences in particular, which have for a long time been puzzled by the seemingly irregular activity of the nervous system. A long series of experiments has now firmly established that sensory neurons of various modalities benefit from ambient noise [3 9].

Theoretical efforts to explain stochastic resonance in neuronal systems had to abstract rigorously from the biological complexity of real neurons to facilitate mathematical treatment 10 14]. The leaky integrate-and-fire model neuron (LIF) 115 is likely the most widely studied of these abstract neuron models, especially in investigations of the neuronal code [16 21]. The main advantages of this model are its simplicity and lack of memory: Each time the neuron has been excited sufficiently to fire a spike (output pulse), it is reset to a predefined state, erasing all memory of past input. One may thus analyze the intervals between spikes separately, and build the complete spike train fired by the neuron by concatenation of intervals.

Time-dependent stimulation complicates this procedure, though, as a different stimulus is now presented during each interspike interval. This precludes a straightforward analysis of global properties of the spike train, such as its power spectral density. Since stochastic reso-

${ }^{*}$ Corresponding author: hans.plesser@itf.nlh.no nance is typically defined in terms of the signal-to-noise ratio in response to periodic stimulation, either of the following is required: (i) further simplification of the model, or (ii) the development of better techniques.

The latter is certainly preferable and has been achieved in the meantime 222 24]. Stochastic resonance with respect to both noise amplitude and signal frequency has been found, and may be relevant to signal processing in neuronal networks [25]26]. Results obtained for the leaky integrate-and-fire neuron are in qualitative agreement with those obtained from more realistic, non-linear models [27.28], indicating that the abstraction to the LIF model was acceptable.

Albeit stochastic resonance in the leaky integrate-andfire model may thus be considered a solved problem, we would like to return here to some earlier studies [29 31,23, which, at least in parts, followed the simplifying approach (i). These studies assumed that the same stimulus was presented during each interspike interval, i.e., that the originally periodic stimulus was reset to a fixed phase $\phi_{0}$ after each spike. All intervals are thus equivalent, and global properties of the spike train may be computed from the interspike-interval distribution for a single interval using the methods of renewal theory [32]. Lánský coined the terms endogenous and exogenous stimulation for stimulation with and without reset, respectively [33].

This stimulus-reset assumption is patently unbiological for virtually all neurons, since it would require the neuron to fully control the input it receives. The reset assumption was therefore justified as an approximation to the full, exogenous dynamics of a periodically driven noisy neuron along the following lines [34]: If the neuron is driven by a periodic subthreshold stimulus (a stimulus too weak to evoke spikes in the absence of noise), then the neuron will fire all spikes at approximately the same stimulus phase $\phi^{*}$ after transients have died out. This phase corresponds roughly to the phase at which the membrane potential is maximal in the absence of noise. Such firing patterns are found in sensory neurons, e.g., in the auditory nerve [35] or cold-receptor neurons [36, 37]. Thus, roughly the same stimulus, starting from phase $\phi(\tau=0) \approx \phi^{*}$, is presented during each interval. Resetting the stimulus phase to a fixed phase $\phi(\tau=0)=\phi_{0}$ will therefore introduce only minor errors.

This reasoning suffers from an essential shortcoming, namely the choice of a fixed reset phase $\phi_{0}$ independent of both stimulus and noise. This reset phase is a free 
parameter of the model, which has no counterpart in biological neurons. We show here that stochastic resonance in terms of the signal-to-noise ratio as studied previously in the LIF model with stimulus reset 30,31,23 occurs because the model neuron adapts best to the free parameter "reset phase" for a particular noise amplitude. The signal-to-noise ratio as defined in those studies diverges monotonically for vanishing noise if the reset phase is adapted to stimulus and noise in a plausible way.

The leaky integrate-and-fire model and the methods applied in analysis are briefly reviewed in Sec. II; see Ref. 26] for details. Results are presented in Sec. III] and summarized in Sec. IV.

\section{MODEL AND METHODS}

\section{A. Leaky integrate-and-fire neuron}

The leaky integrate-and-fire neuron model sketches the neuron as a capacitor with leak current, which is charged by an input current $I(t)$ until the potential $v(t)$ across the capacitor (membrane potential) reaches a threshold $\Theta$. At that instant, an output spike is recorded and the potential reset to $v_{r}<\Theta$. The assumption that the stimulus is reset after each spike as well implies that the membrane potential evolves according to

$$
\dot{v}(\tau)=-v(\tau)+\mu+q \cos \left(\Omega \tau+\phi_{0}\right)+\sigma \xi(\tau),
$$

in between any two spikes 15]; $\tau$ is intra-interval time, i.e., $\tau$ runs from zero in every interval. $\mu$ is the $\mathrm{DC}$ component of the stimulus, $q$ its $\mathrm{AC}$ amplitude, $\Omega$ the nominal frequency, and $\phi_{0}$ the fixed but arbitrary reset phase. All quantities are measured in their natural units, i.e., the membrane time constant $\tau_{m}$ and threshold $\Theta$. $v_{r}=0$ is assumed throughout. The noise term in Eq. (11) subsumes both biochemical and network noise [38, 39, and is taken to be Gaussian white noise $\left[\left\langle\xi(t) \xi\left(t^{\prime}\right)\right\rangle=\right.$ $\left.\delta\left(t-t^{\prime}\right)\right]$. A different realization of the noise is used for each interval. Sub-threshold stimuli are characterized by $\sup _{t \rightarrow \infty} v(t)=\mu+q / \sqrt{1+\Omega^{2}}<1$ for $\sigma=0$. We restrict ourselves here to these stimuli, because they appear to be more relevant for the encoding of periodic signals in sensory systems than superthreshold stimuli [16,40].

The sequence $\tau_{1}, \tau_{2}, \ldots, \tau_{k}, \ldots$ of intervals corresponds to an output spike train $f(t)=\sum_{k} \delta\left(t-t_{k}\right)$ with spike times $t_{k}=\sum_{j \leq k} \tau_{j}$. This spike train is evoked by an effective stimulus consisting of piecewise sinusoids of length $\tau_{k}$, as shown in Fig. 1. In contrast, we call the pure sinusoid $\cos \left(\Omega t+\phi_{0}\right)$ the nominal stimulus. Figure 1 indicates that the effective stimulus approximates the nominal stimulus for a reset phase of $\phi_{0} \approx 0$, while it is an irregular sequence of piecewise sinusoids for other choices of the reset phase. We therefore focus here on $\phi_{0}=0$ in accordance with earlier work $30,31,23$.
Because of the stimulus reset and the whiteness of the noise, all interspike interval lengths $\tau_{k}$ are statistically independent, identically distributed random variables with density $\rho(\tau)$. The latter can be computed numerically or approximated in closed form [26, 41, 42, The sequence of intervals thus forms a renewal process, which is fully characterized by the ISI density $\rho(\tau)$ 43. Periodic subthreshold stimulation evokes multimodal ISI densities as shown in Fig. 2 for noise not too strong. The location of the first peak depends on the reset phase $\phi_{0}$, while subsequent peaks follow at intervals of the nominal stimulus period $T=2 \pi / \Omega$. Comparable ISI distributions are found in sensory neurons 35,36,44.

\section{B. Signal-to-noise ratio}

The performance of a signal processor is commonly measured in terms of the signal-to-noise ratio in studies on stochastic resonance. Since the spike train elicited from the neuron is a renewal process by virtue of the stimulus reset, its power spectral density (PSD) is given by 32,30

$$
S(\omega)=\frac{1}{\pi\langle\tau\rangle}\left(1+2 \Re \frac{\tilde{\rho}(\omega)}{1-\tilde{\rho}(\omega)}\right), \omega>0,
$$

where

$$
\tilde{\rho}(\omega)=\int_{0}^{\infty} \rho(\tau) e^{i \omega \tau} d \tau
$$

is the Fourier transform of the ISI density and $\langle\tau\rangle$ the mean interspike interval length; note that $\rho(\tau)=0$ for $\tau<0$ by definition.

The input to the neuron is not purely sinusoidal due to the stimulus reset, and the maximum of the PSD will thus be shifted away from the stimulus frequency $\Omega$, see Fig. 3(a). We thus define the signal as the maximum of the PSD in a window around $\Omega$ [30, 31

$$
\hat{S}=S(\hat{\Omega})=\max \{S(\omega) \mid 0.9 \Omega<\omega<1.1 \Omega\}
$$

and refer to the location $\hat{\Omega}$ of the maximum as peak frequency. The signal $\hat{S}$ is undefined if $S(\omega)$ has no absolute maximum within the window as, e.g., in Fig. 3(b). The white power spectrum of a Poissonian spike train of equal intensity, $S_{P}=(\pi\langle\tau\rangle)^{-1}$, is used as reference noise level [45], whence the signal-to-noise ratio is

$$
R_{\mathrm{SN}}=\hat{S} / S_{p}=\pi\langle\tau\rangle \hat{S}
$$

Note that power spectral density and signal-to-noise ratio as defined above are calculated for infinitely long spike trains. Any strictly periodic component of the spike train will thus give rise to singularities in the PSD. In the reset model, coherence is broken by the stimulus reset, resulting in a continuous spectrum 46,26. Due to the different definitions, signal-to-noise ratios obtained from 
the LIF model with and without stimulus reset cannot be compared quantitatively 23.

The spectrum defined by Eq. (2) may have very narrow peaks for low noise, whence numerical evaluation of Eqs. (2) -(5) may require very high frequency resolution.

\section{Preferred firing phase}

If the stimulus is not reset after each spike, the probability $\chi^{(k+1)}(\psi)$ for the $k+1$-st spike to occur at stimulus phase $\psi$ can be expressed in terms of the corresponding distribution for the $k$-th spike as

$$
\chi^{(k+1)}(\psi)=\int_{-\pi}^{\pi} \mathcal{T}(\psi \mid \phi) \chi^{(k)}(\phi) d \phi
$$

where $\mathcal{T}(\psi \mid \phi)$ is a stochastic kernel 47,24. For $k \rightarrow \infty$, the firing phase distribution will approach a stationary distribution $\chi^{(s)}$ and we choose the preferred firing phase as the phase at which the neuron most likely fires,

$$
\phi^{*}=\arg \max _{\psi} \chi^{(s)}(\psi)
$$

Using this preferred phase as reset phase will yield a viable approximation to stimulation without reset only if the firing phase distribution is sharply concentrated around $\phi^{*}$, and has only a single maximum. To ensure this, we require that the vector strength 48 of the distribution fulfills

$$
r=\left|\left\langle e^{i \phi}\right\rangle\right|=\left|\int_{-\pi}^{\pi} e^{i \phi} \chi^{(s)}(\phi) d \phi\right| \geq 0.9 .
$$

This condition is generally met by the sub-threshold stimuli combined with weak noise studied here. Multimodal firing phase distributions are observed only for slow stimuli in the presence of intermediate to large noise, and for superthreshold stimuli. We are therefore not concerned with complications that may arise from modelocking as observed in the latter cases 49.52 .

\section{RESULTS}

\section{A. Fixed Reset Phase}

For fixed reset phase, $\phi_{0}=0$, the model neuron shows typical stochastic resonance behavior, i.e., a maximum of the SNR at an intermediate albeit small noise amplitude $\sigma_{\max }$ as shown in Fig. 4(a) [30]. The mechanism inducing stochastic resonance is indicated in Fig. A(b): the maximal SNR is reached when the peak frequency $\hat{\Omega}$ and the reset frequency $\Omega_{\text {res }}=2 \pi / T_{\text {res }}$ coincide, where $T_{\text {res }}$ is the mode of the ISI density, i.e., the most probable interval between two stimulus resets. Coincidence of reset and peak frequencies thus indicates synchronization between the stimulus reset and the correlations dominating the power spectrum of the output spike train, yielding optimal encoding of the nominal stimulus.

This effect may intuitively be explained as follows. Assume that the peaks of the ISI distribution are located around $\tau=n T+\epsilon, \epsilon>0$, for a given choice of reset phase and small noise. The effective stimulus will then be a close to the nominal stimulus, cf. Fig. 11(a). The neuron will fire at shorter intervals as the noise is increased, and for a particular noise amplitude will the peaks of the ISI distribution be centered about multiples of the stimulus period $T$. The effective stimulus will then be reset in intervals of $n T$. The neuron is thus on average driven by a periodic stimulus with period $\Omega_{\text {res }}=\Omega$, evoking as periodic a spike train as possible, i.e., one maximizing the signal-to-noise ratio. This explanation ignores all jitter in spike timing, which reduces the SNR. The SNR maximum is thus not found for that input noise amplitude which yields $\Omega_{\text {res }}=\Omega(\sigma \approx 0.018)$, but for somewhat weaker input noise, corresponding to smaller output jitter, cf. Fig. 4(b).

\section{B. Noise-Adapted Reset Phase}

A LIF neuron driven by a sinusoidal stimulus which is not reset after each spike will approach a stationary firing pattern 47,24. The preferred firing phase in the stationary state is given by Eq. (7) and depends on the noise amplitude as shown in Fig. 5(c): the neuron fires at later phases for weaker noise. Note that interspike intervals will be multiples of the stimulus period $T$ in this regime, since the neuron fires all spikes at the same phase $\phi^{*}$ (up to jitter).

This observation suggests how to construct a proper approximation to the full LIF dynamics using the reset model: for each stimulus and each noise amplitude, determine the preferred phase from Eq. (7) and use this phase as reset phase,

$$
\phi_{0}=\phi_{0}(\sigma ; \mu, q, \Omega)=\phi^{*}(\sigma ; \mu, q, \Omega)
$$

The stimulus is then reset in intervals of multiples of the stimulus period, so that the effective stimulus will differ from the nominal stimulus only through jitter. This jitter vanishes as input noise vanishes, whence perfect periodic stimulation will be attained for $\sigma \rightarrow 0$. Consequently, the signal-to-noise ratio will diverge for vanishing noise as shown in Fig. 5(a). The reset frequency is identical to the nominal frequency by construction, $\Omega_{\text {res }}=\Omega$, cf. Fig. 5(b). The peak frequency, on the other hand, converges to the stimulus frequency as noise vanishes, $\hat{\Omega} \rightarrow \Omega_{\text {res }}=\Omega$, as the effective stimulus becomes identical to the nominal one.

We shall now make this argument rigorous. The ISI distribution of a LIF model neuron is well approximated by 42

$$
\rho(\tau) \approx h(\tau) \exp \left[-\int_{0}^{\tau} h(s) d s\right]
$$




$$
h(\tau)=\exp \left[-\left(\frac{1-v_{0}(\tau)}{\sigma}\right)^{2}\right]
$$

where $v_{0}(\tau)$ is the membrane potential in the absence of noise, i.e., the solution of Eq. (11) for $\sigma=0$. We consider the limit of small noise $(\sigma \ll 1)$ and slow stimulation $(T \gg 1)$. In that case, we can neglect transient terms in the membrane potential to obtain

$$
v_{0}(\tau)=\mu+\hat{q} \cos (\Omega \tau+\Omega \zeta)+\mathcal{O}\left(e^{-\tau}\right),
$$

where $\hat{q}=1 / \sqrt{1+\Omega^{2}}$, and $\zeta=\left(\phi_{0}-\arctan \Omega\right) / \Omega$. The hazard $h(\tau)$ will thus be a sequence of narrow peaks around the maxima of $v_{0}(\tau)$, i.e. around $\tau_{n}=n T-\zeta$. ISI lengths are multiples of the stimulus period for adapted reset phase by construction, whence $\zeta=0$ for $\phi_{0}=$ $\phi^{*}(\sigma)$.

As peaks are narrow, we may take the exponential term in Eq. (10) to be constant across each peak; it merely reduces the peak amplitude by a factor $\gamma$ for each subsequent peak. The ISI distribution can thus be approximated as a superposition of dampened copies of the hazard function centered about $\tau_{n}=n T$, i.e.,

$$
\rho(\tau) \approx \sum_{n=1}^{\infty} \gamma^{n-1} \exp \left[-\left(\frac{1-v_{0}\left(\tau-\tau_{n}\right)}{\sigma}\right)^{2}\right]
$$

with $\gamma=\exp \left(-\int_{T / 2}^{3 T / 2} h(s) d s\right)$. Expanding the exponent separately for each summand and retaining only terms of lowest order in $\tau-\tau_{n}$ yields,

$$
\rho(\tau) \approx c \sum_{n=1}^{\infty} \gamma^{n-1} \exp \left(-\frac{\Omega^{2}(\tau-n T)^{2}}{\eta \sigma^{2}}\right),
$$

where $\eta^{-1}=\hat{q}(1-\mu-\hat{q})$, and $c$ is a normalization factor. The interspike-interval distribution has thus been reduced to a sum of Gaussians. This approximation holds well for small noise, as shown in Fig. 6.

The Fourier transform of the ISI distribution is thus

$$
\tilde{\rho}(\omega)=\frac{(1-\gamma) \exp \left[-\frac{\eta \sigma^{2}}{4}\left(\frac{\omega}{\Omega}\right)^{2}+2 \pi i \frac{\omega}{\Omega}\right]}{1-\gamma \exp \left[2 \pi i \frac{\omega}{\Omega}\right]} .
$$

In the limit of small noise and for adapted reset phase, the effective stimulus becomes equal to the nominal stimulus, and the spectral power will be maximal at the nominal stimulus frequency in this limit, i.e., $\hat{\Omega} \rightarrow \Omega$ for $\sigma \rightarrow 0$, cf. Fig. F(b). The signal-to-noise ratio is therefore

$$
R_{\mathrm{SN}}=\frac{S(\Omega)}{S_{P}} \approx \operatorname{coth} \frac{\eta \sigma^{2}}{8} \quad(\sigma \ll 1)
$$

in good agreement with numerical results for the exact model (solid line in Fig. 5(a)). We find in particular that the signal-to-noise ratio diverges for vanishing noise in the Gaussian approximation.

\section{CONCLUSIONS}

The aim of this paper was to clarify whether the response of the leaky integrate-and-fire neuron to periodic subthreshold stimulation can be approximated as a renewal process. In particular, we wanted to know whether stochastic resonance at weak noise as reported in earlier work [30,23] is a genuine property of the LIF neuron, or rather an artefact of the stimulus reset, which was introduced to reduce the full neuronal dynamics to a renewal process.

We argued that renewal (endogenous) dynamics are a good approximation to the full (exogenous) dynamics only if the phase $\phi_{0}$, to which the stimulus is reset after each spike, is adapted to the stimulus parameters, especially the noise amplitude, in such a way that the neuron fires most likely at phase $\phi_{0}$. We showed that stochastic resonance does not occur in this case. Stochastic resonance is only found if the reset phase is held fixed as the amplitude of the input noise is varied.

There is no biologically plausible way in which a neuron could reset a stimulus impinging on it to a fixed phase. Lánský suggested that neurons driven by internally generated membrane potential oscillations could reset their oscillator to a fixed phase, hence the term endogenous stimulation [33]. Cold receptor cells are driven by internal subthreshold oscillations, but do not reset their internal oscillator upon firing [37]. It is thus highly unlikely to find neurons which reset the oscillator that drives them to a fixed phase independent of stimulus properties, i.e., follow genuine endogenous dynamics. This in turn means that any effect arising solely from the reset to a fixed stimulus phase will not be found in real neurons. The stochastic resonance effect reported in Ref. [30] (see also 23]) is thus a model artefact.

We stress that this conclusion applies only to the particular type of stochastic resonance discussed here. The leaky integrate-and-fire neuron benefits from stochastic resonance when encoding periodic signals without reset (exogenous stimulation), as shown in [22,24,23]. This resonance occurs at noise amplitudes which are about one order of magnitude larger than the resonance found in the model with reset to a fixed phase. The crucial difference is that studies on exogenous stimulation consider either explicitly or implicitly the power spectral density of a spike train of finite duration, while an infinite spike train is assumed here, cf. Sec. IIB. Trains of very low intensity, but precisely phase-locking to the stimulus, as found for very weak noise, yield a small finite-time signal-to-noise ratio, while their infinite-time SNR may be large.

Other noise-induced resonance phenomena found in the LIF with stimulus reset, e.g., in the interspikeinterval distribution or the mean ISI length 29, 34], are not directly affected by our finding either. Indeed, Shimokawa et al. found comparable resonance effects in terms of the ISI distribution for stimulation with and without reset 22]. These resonances occur at much larger 
noise amplitudes than studied here; see also Ref. 23.

Our findings should, on the other hand, not be restricted to the particular neuron model studied here. We expect that stochastic resonance may be introduced to nearly any threshold system when the full dynamics under periodic forcing are reduced to a renewal process by resetting the forcing to a noise-independent phase after each threshold crossing. Periodic forcing may then be recovered by adjusting the noise such that threshold crossings occur around the predefined reset phase, whence periodic forcing is recovered, and optimal output attained. Unless the fixed reset phase has a counterpart in the physical system under study, this resonance is obviously an artefact of a simplification carried too far.

\section{ACKNOWLEDGMENTS}

We would like to thank G. T. Einevoll for critically reading an earlier version of the manuscript and two anonymous referees for helpful comments. HEP is supported by an EU Marie-Curie-Fellowship.

[1] L. Gammaitoni, P. Hänggi, P. Jung, and F. Marchesoni, Rev. Mod. Phys. 70, 223 (1998).

[2] K. Wiesenfeld and F. Jaramillo, Chaos 8, 539 (1998).

[3] A. Longtin, A. Bulsara, and F. Moss, Phys. Rev. Lett. 67, 656 (1991).

[4] J. K. Douglass, L. Wilkens, E. Pantazelou, and F. Moss, Nature (London) 365, 337 (1993).

[5] J. E. Levin and J. P. Miller, Nature (London) 380, 165 (1996).

[6] J. J. Collins, T. T. Imhoff, and P. Grigg, J. Neurophysiol. 76, 642 (1996).

[7] P. Cordo et al., Nature (London) 383, 769 (1996).

[8] F. Jaramillo and K. Wiesenfeld, Nature Neurosci. 1, 384 (1998).

[9] D. F. Russell, L. A. Wilkens, and F. Moss, Nature (London) 402, 291 (1999).

[10] T. Zhou, F. Moss, and P. Jung, Phys. Rev. A 42, 3161 (1990).

[11] A. Bulsara et al., J. Theor. Biol. 152, 531 (1991).

[12] Z. Gingl, L. B. Kiss, and F. Moss, Europhys. Lett. 29, 191 (1995).

[13] K. Wiesenfeld et al., Phys. Rev. Lett. 72, 2125 (1994).

[14] J. J. Collins, C. C. Chow, A. C. Capela, and T. T. Imhoff, Phys. Rev. E 54, 5575 (1996).

[15] H. C. Tuckwell, Stochastic Processes in the Neurosciences (SIAM, Philadelphia, 1989).

[16] W. Gerstner, R. Kempter, J. L. van Hemmen, and H. Wagner, Nature (London) 383, 76 (1996).

[17] P. Maršálek, C. Koch, and J. Maunsell, Proc. Natl. Acad. Sci. USA 94, 735 (1997).
[18] T. W. Troyer and K. D. Miller, Neural Comput. 9, 971 (1997).

[19] G. Bugmann, C. Christodoulou, and J. G. Taylor, Neural Comput. 9, 985 (1997).

[20] J. Feng, Phys. Rev. Lett. 79, 4505 (1997).

[21] L. F. Abbott, J. A. Varela, K. Sen, and S. B. Nelson, Science 275, 220 (1997).

[22] T. Shimokawa, A. Rogel, K. Pakdaman, and S. Sato, Phys. Rev. E 59, 3461 (1999).

[23] T. Shimokawa, K. Pakdaman, and S. Sato, Phys. Rev. E 60, R33 (1999).

[24] H. E. Plesser and T. Geisel, Phys. Rev. E 59, 7008 (1999).

[25] H. E. Plesser and T. Geisel, Signal Processing by Means of Noise, Submitted, 2000.

[26] H. E. Plesser, Ph.D. thesis, Georg-August-Universität, Göttingen, 1999.

[27] F. Liu, J. F. Wang, and W. Wang, Phys. Rev. E 59, 3453 (1999).

[28] T. Kanamaru, T. Horita, and Y. Okabe, Phys. Lett. A 255, 23 (1999).

[29] A. R. Bulsara et al., Phys. Rev. E 53, 3958 (1996).

[30] H. E. Plesser and S. Tanaka, Phys. Lett. A 225, 228 (1997).

[31] T. Shimokawa, K. Pakdaman, and S. Sato, Phys. Rev. E 59, 3427 (1999).

[32] J. Franklin and W. Bair, SIAM J. Appl. Math. 55, 1074 (1995).

[33] P. Lánský, Phys. Rev. E 55, 2040 (1997).

[34] A. R. Bulsara, S. B. Lowen, and C. D. Rees, Phys. Rev. E 49, 4989 (1994).

[35] J. E. Rose, J. F. Brugge, D. J. Anderson, and J. E. Hind, J. Neurophysiol. 30, 769 (1967).

[36] H. A. Braun, K. Schäfer, and H. Wissing, Funkt. Biol. Med. 3, 26 (1984).

[37] A. Longtin and K. Hinzer, Neural Comput. 8, 215 (1996).

[38] A. Manwani and C. Koch, Neural Comput. 11, 1797 (1999).

[39] Z. F. Mainen and T. J. Sejnowski, Science 268, 1503 (1995).

[40] R. Kempter, W. Gerstner, J. L. van Hemmen, and H. Wagner, Neural Comput. 10, 1987 (1998).

[41] A. Buonocore, A. G. Nobile, and L. M. Ricciardi, Adv. Appl. Prob. 19, 784 (1987).

[42] H. E. Plesser and W. Gerstner, Neural Comput. 12, 367 (2000).

[43] D. R. Cox and H. D. Miller, The Theory of Stochastic Processes (Methuen\&Co, London, 1965).

[44] R. A. Lavine, J. Neurophysiol. 34, 467 (1971).

[45] M. Stemmler, Network 7, 687 (1996).

[46] M. B. Priestley, Spectral Analysis and Time Series (Academic Press, London, 1996).

[47] T. Tateno, S. Doi, S. Sato, and L. M. Ricciardi, J. Stat. Phys. 78, 917 (1995).

[48] J. M. Goldberg and P. B. Brown, J. Neurophysiol. 32, 613 (1969).

[49] S. Coombes and P. C. Bressloff, Phys. Rev. E 60, 2086 (1999).

[50] J. D. Hunter, J. G. Milton, P. J. Thomas, and J. D. Cowan, J. Neurophysiol. 80, 1427 (1998).

[51] T. Tateno, J. Stat. Phys. 92, 675 (1998). 

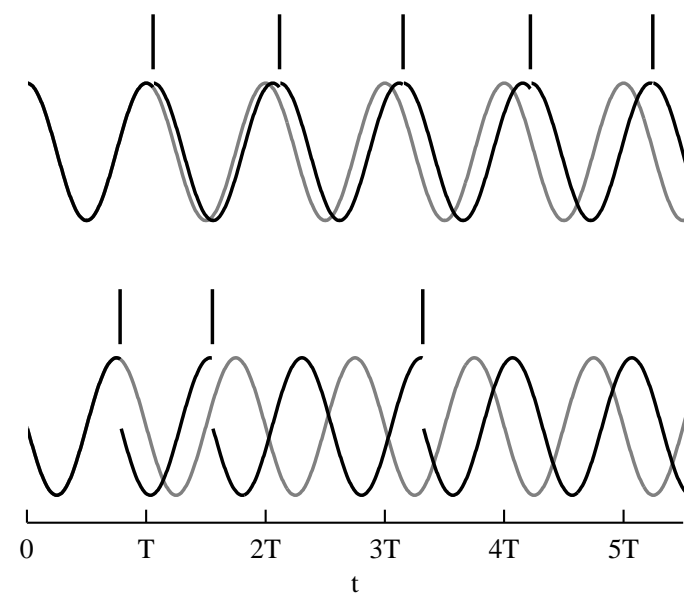

FIG. 1. Effective stimulus and corresponding spike trains for fixed reset phases $\phi_{0}=0$ (top) and $\phi_{0}=\pi / 2$ (bottom) are shown in black, while the nominal stimuli are shown in grey. The reset has small consequences in the first case, while the effective stimulus differs markedly from the nominal sinusoid for the latter. $T=2 \pi / \Omega$ is the period of the nominal stimulus; amplitudes are in arbitrary units. Remaining stimulus parameters: $\mu=0.9, q=0.1, \Omega=0.1 \pi, \sigma=0.008$.

[52] F. C. Hoppensteadt and J. P. Keener, J. Math. Biology 15, 339 (1982).

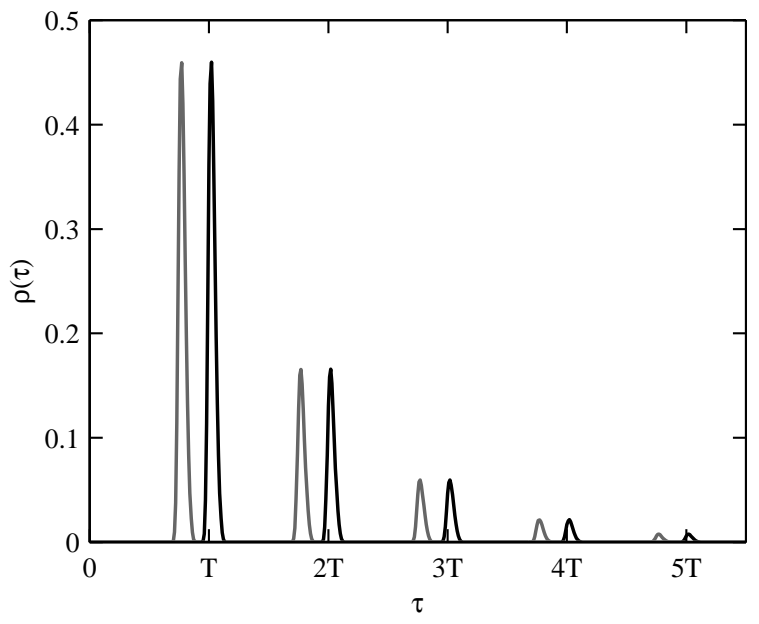

FIG. 2. Interspike-interval distribution in response to periodic stimulation with reset to phases $\phi_{0}=0$ (black) and $\phi_{0}=\pi / 2$ (grey). All else as in Fig. 1.
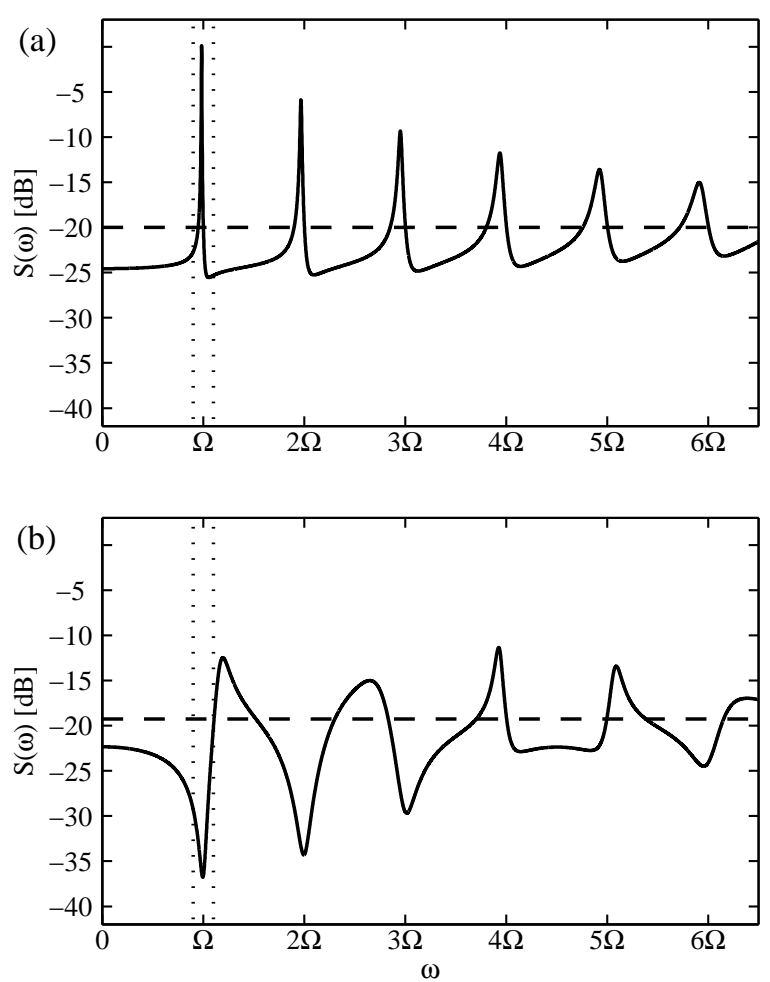

FIG. 3. Power spectral density for (a) reset phase $\phi_{0}=0$, and (b) $\phi_{0}=\pi / 2$. The dashed horizontal line is the PSD $S_{P}$ of a Poisson train of equal intensity; vertical dotted lines mark the interval $[0.9 \Omega, 1.1 \Omega]$, cf. Eq. (四). Note the lack of power in the vicinity of the nominal stimulus frequency $\Omega$ for reset phase $\phi_{0}=\pi / 2$. All else as in Fig. 1 . 

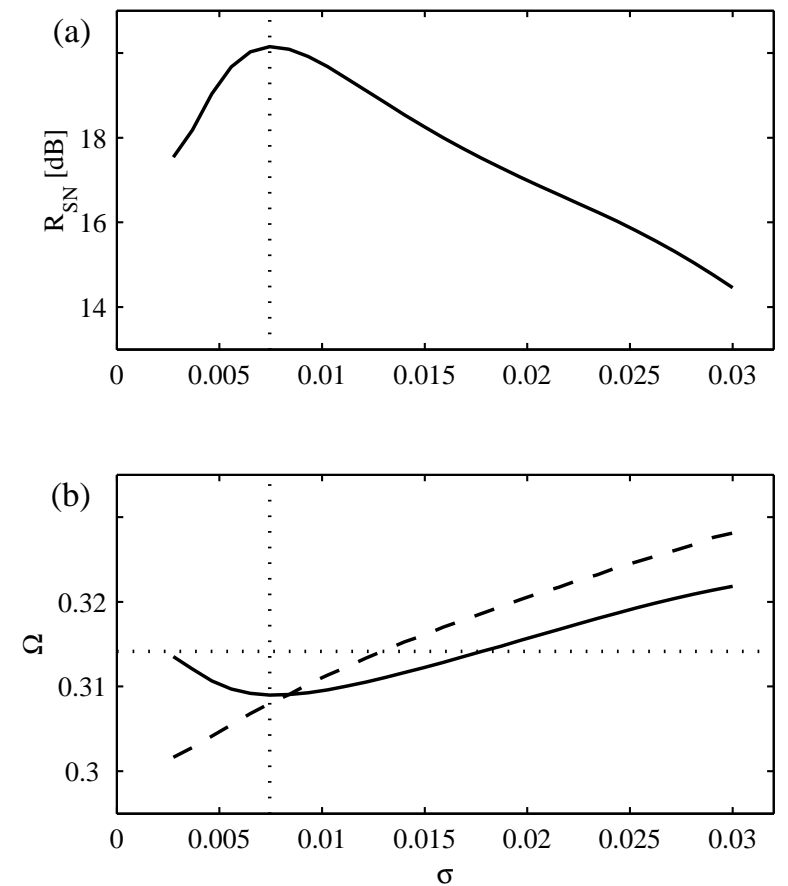

FIG. 4. (a) Signal-to-noise ratio vs. input noise amplitude for fixed reset phase $\phi_{0}=0$. (b) Peak frequency $\hat{\Omega}$ (solid) and reset frequency $\Omega_{\text {res }}$ (dashed) vs. noise amplitude for fixed reset phase. The dotted horizontal line marks the nominal stimulus frequency $\Omega=0.1 \pi$, while the dotted vertical line marks the optimal noise amplitude. Stimulus parameters as in Fig. 回.
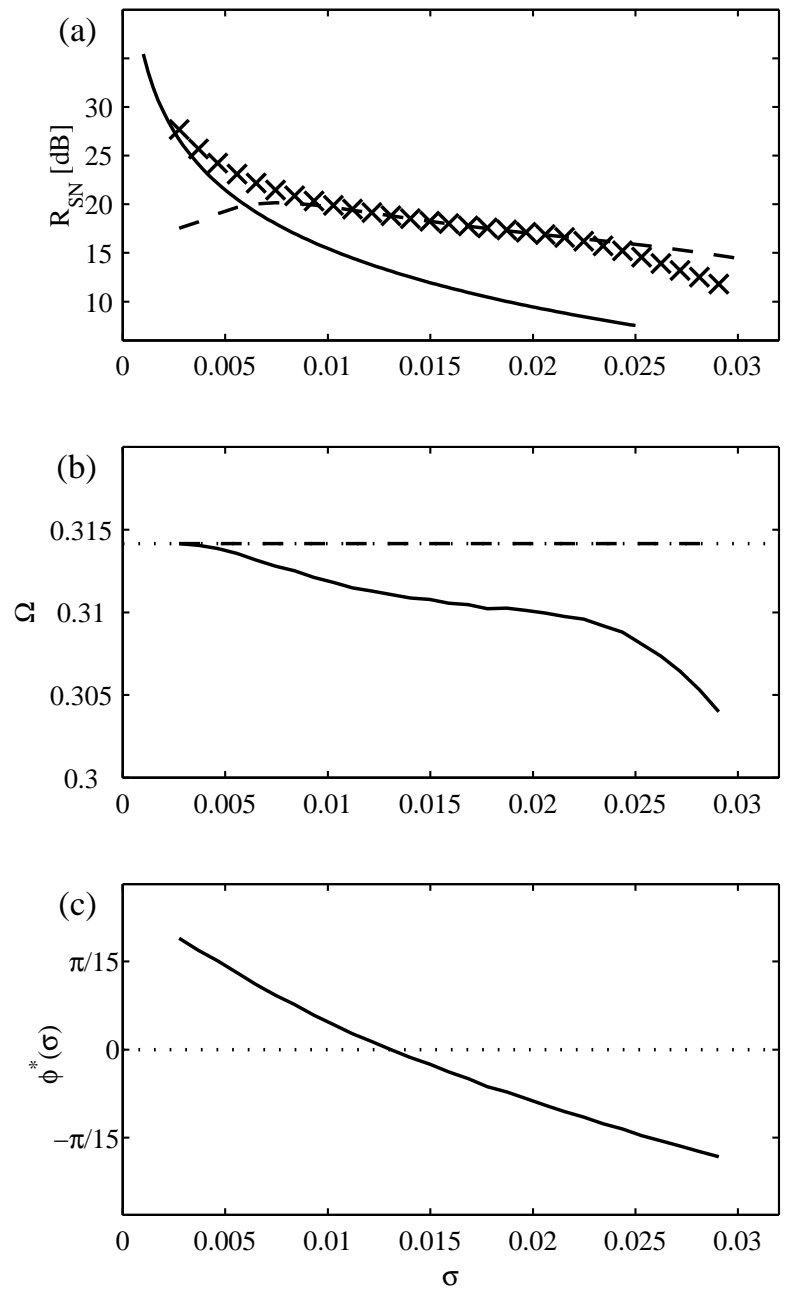

FIG. 5. (a) Signal-to-noise ratio vs. input noise amplitude for adapted reset phase $\phi_{0}=\phi^{*}(\sigma)$ (symbols) and for fixed reset phase $\phi_{0}=0$ for comparison (dashed). The solid line is the approximation of Eq. (16). (b) Peak frequency $\hat{\Omega}$ (solid) and reset frequency $\Omega_{\text {res }}$ (dashed) vs. noise amplitude for fixed reset phase. The dotted line marks the nominal stimulus frequency. (c) Preferred frequency $\phi^{*}(\sigma)$ vs. noise. Stimulus parameters as in Fig. 1.

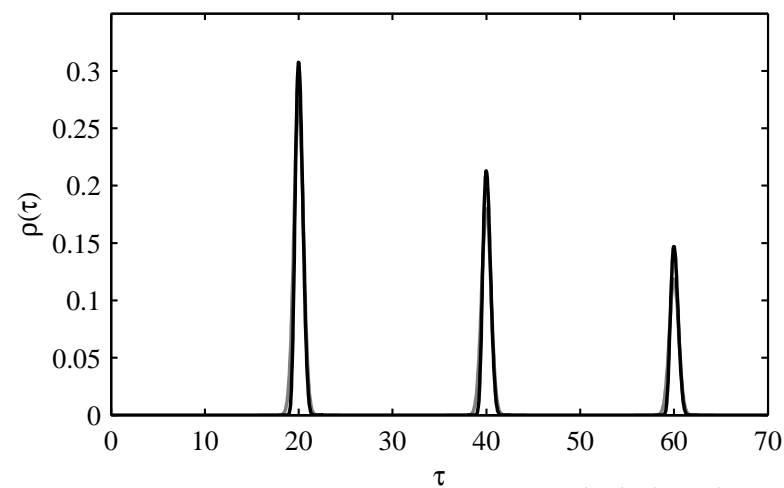

FIG. 6. Gaussian approximation of Eq. (14) (grey) to the interspike-interval distribution (black) for the same stimulus parameters as before and $\sigma=0.0046$. 